Ref. SISSA 94/96/FM

\title{
A Note on Fractional KdV Hierarchies
}

\author{
Paolo Casati $^{1}$, Gregorio Falqui ${ }^{2}$, \\ Franco Magri ${ }^{1}$, and Marco Pedroni ${ }^{3}$ \\ 1 Dipartimento di Matematica, Università di Milano \\ Via C. Saldini 50, I-20133 Milano, Italy \\ E-mail: casati@vmimat.mat.unimi.it, magri@vmimat.mat.unimi.it \\ 2 SISSA/ISAS, Via Beirut 2/4, I-34014 Trieste, Italy \\ E-mail: falqui@sissa.it \\ 3 Dipartimento di Matematica, Università di Genova \\ Via Dodecaneso 35, I-16146 Genova, Italy \\ E-mail: pedroni@dima.unige.it
}

\begin{abstract}
One of the cornerstone of the theory of integrable systems of KdV type has been the remark that the $n$-GD equations are reductions of the full KP theory. In this paper we address the analogous problem for the fractional KdV theories, by suggesting candidates of the "KP-theories" lying behind them. These equations are called "KP( ${ }^{(m)}$ hierarchies", and are obtained as reductions of a bigger dynamical system, which we call the "central system". The procedure allowing to pass from the central system to the $\mathrm{KP}^{(m)}$ equations, and then to the fractional $\mathrm{KdV}_{n}^{m}$ equations, is discussed in detail in the paper. The case of $\mathrm{KdV}_{3}^{2}$ is given as paradigmatic example.

PACS numbers: 02.40, 03.20

Work supported by the Italian M.U.R.S.T. and by the G.N.F.M. of the Italian C.N.R.

One of the authors (F.M.) thanks the organizers of the Semester on Integrable Systems for the kind hospitality at the Centre Émile Borel, where part of this work has been completed
\end{abstract}




\section{Introduction}

The study of integrable systems of KdV type received, in the last few years, a new impulse from important developments in Two-Dimensional (Quantum) Field Theory. In this framework, much attention has been paid to the conformal $\mathcal{W}_{N}$-algebras of symmetries of these theories. In the cases studied at first, they have been shown [1, 2] to be quantum extension of the second Pois-

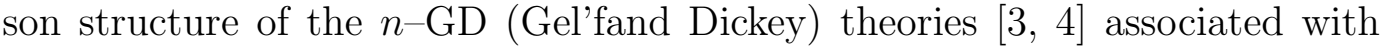
a classical Lie algebra $\mathfrak{g}$. The powerful method of Hamiltonian reduction has been widely applied to study and classify those hierarchies of partial differential equations and the associated $\mathcal{W}$-algebras (see, e.g., [5]). A class of new integrable hierarchies (called fractional KdV or generalized DS) has been obtained [6, 0, 8, 9, 10, 11] by means of a generalization of the Drinfel'd-Sokolov construction. Roughly speaking, fractional KdV hierarchies correspond to the case in which the value of the momentum mapping for the infinitesimal gauge action of the loop algebra $L \mathfrak{g}$ is different from the sum of the (duals of the) simple positive roots of $\mathfrak{g}$. They have been shown [8, 9, 12] to be classified by homogeneous elements in Heisenberg subalgebras of the affinization $\tilde{\mathfrak{g}}$ of $\mathfrak{g}$.

One of the cornerstones of the theory of integrable systems of KdV type is the fact that $n$-GD theories can be obtained from the full KadomtsevPetviashvilij (KP) hierarchy by means of a suitable reduction process [ 4 , 13, 14. In this paper we study the problem of the generalization of such a link to the case of fractional KdV theories. Namely we want to suggest candidates of "KP-theories" lying behind fractional KdV ones.

Our starting point is a special hierarchy of dynamical systems described by equations of Riccati type, which we call the central system (CS). They are defined on the space $\mathcal{H}$ of sequences $\left\{H^{(k)}\right\}_{k \in \mathbb{N}}$ of Laurent series of the form

$$
H^{(0)}=1, \quad H^{(k)}=z^{k}+\sum_{l \geq 1} H_{l}^{k} z^{-l}
$$

by the following equations

$$
\frac{\partial H^{(k)}}{\partial t_{j}}+H^{(j)} H^{(k)}=H^{(j+k)}+\sum_{l=1}^{k} H_{l}^{j} H^{(k-l)}+\sum_{l=1}^{j} H_{l}^{k} H^{(j-l)} .
$$

They arise in the framework of the bihamiltonian approach to the KdV equation, as we shall see in Section 2. One can remark that the space $\mathcal{H}$ is a subspace of the big cell of the Sato Grassmannian $\operatorname{Gr}(H)$ [15, 13], and there-

fore, one can suspect that these equations are related to the linear flows of the $g l_{\infty}$-action on $\operatorname{Gr}(H)$. This is indeed correct, since one can prove that the two types of equations are related by a Darboux transformations. The study of this connection, however, is outside the scopes of the present paper [16]. Our aim, in this paper, is to derive the fractional KdV equations from CS by a double process of reduction. 
The ideas of the present approach are conveniently described by adopting a geometric point of view. We regard equations (1.2) as defining a hierarchy of vector fields $X_{j}$ on $\mathcal{H}$. We notice that these vector fields commute, and we consider the space

$$
\mathcal{Q}^{m}=\mathcal{H} / X_{m}
$$

of the orbits of the vector field $X_{m}$. Since the CS flows commute, each vector field $X_{j}$ sends solutions of $X_{m}$ into different solutions, and therefore induces a flow on the space $\mathcal{Q}_{m}$. We shall call the hierarchy of the reduced flows on $\mathcal{Q}_{m}$ the $K P^{(m)}$ hierarchy. The reason is that for $m=1$, one obtains in this way the usual KP hierarchy in the $1+1$-dimensional picture. We were led to consider different values of $m$ by the conjecture [10, 25] that $m$-fractional $\mathrm{KdV}$ hierarchies can be obtained by "exchanging the roles of $t_{m}$ and $t_{1}$ " in the $m$-GD equations. However, we stress that the projection onto $\mathcal{Q}_{m}$, which can also be thought of as a field-theoretic redefinition of flows and "independent variables", in our geometrical scheme is more conveniently considered as a reduction of the central system.

Besides the space $\mathcal{Q}^{m}$, we consider the manifold

$$
\mathcal{Z}_{n}:=\left\{H \in \mathcal{H} \mid X_{n}(H)=0\right\}
$$

of the zeroes of $X_{n}$ and its submanifold

$$
\mathcal{S}_{n}=\left\{H \in \mathcal{Z}_{n} \mid H^{(n)}=z^{n}\right\},
$$

where the Laurent series $H^{(n)}$ assumes the constant value $H^{(n)}=z^{n}$. We remark that they are invariant submanifolds for the central system. The restrictions of the vector fields $X_{j}$ to $\mathcal{S}_{n}$ give rise to a second system of reduced equations which we call $C S_{n}$ equations. Finally, we combine the two reduction processes, by considering the space

$$
\mathcal{Q}_{n}^{m}=\mathcal{S}_{n} / X_{m}
$$

of the orbits of the vector field $X_{m}$ restricted to $\mathcal{S}_{n}$. We obtain a reduction of the $\mathrm{CS}_{n}$ equations which we call $K d V_{n}^{m}$ equations. Since the reduction processes commute, they can also be seen as restrictions of the $\mathrm{KP}^{(m)}$ equations to $\mathcal{Q}_{n}^{m}$. The relevant manifolds are shown in Figure 1 .

It is our belief that, when $m$ and $n$ are coprime, the $\mathrm{KdV}_{n}^{m}$ equations coincide with the fractional $\mathrm{KdV}$ equations (or generalized type I GD equations) studied in [8, 12]. This will be shown explicitly in the case of $\mathrm{KdV}_{3}^{2}$.

Other interesting equations can be obtained by different choices of the reduction spaces. So, for example, the restrictions of $X_{j}$ to the intersection $\mathrm{S}_{n} \cap \mathrm{Z}_{p}$ of two invariant manifolds give rise to finite dimensional dynamical 
Figure 1: The Reduced Manifolds

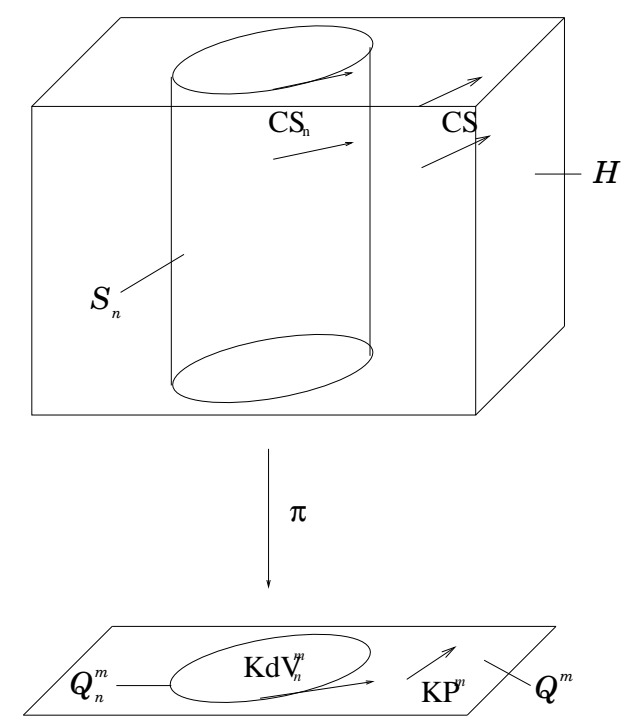

systems of Bogoyavlensky-Dubrovin-Novikov type [17, 18]. Similarly, the projection of the $\mathrm{KP}^{(m)}$ hierarchy into the space

$$
\mathcal{Q}^{(m, l)}=\mathcal{Q}^{m} / X_{l}
$$

of the orbits of the reduction of $X_{l}$ to $\mathcal{Q}^{m}$, can lead to equations in two space dimensions. They are denoted $\mathrm{KP}^{(m, l)}$ in Figure 2, where the full reduction scheme is represented; the left arrows denote a restriction to invariant submanifolds, and the right arrows denote a projection onto orbit spaces $\mathcal{Q}_{m}$.

The main aim of this paper is to study the equations $\mathrm{CS}_{n}, \mathrm{KP}^{(m)}$ and $\mathrm{KdV}_{n}^{m}$ and their relations. The paper is organized as follows. Section 2 is a brief introduction to the central system from the point of view of the bihamiltonian approach to the KdV equations. The first fundamental properties of CS are studied in Section 3, where we describe in detail the submanifolds $\mathcal{S}_{n}$ and the quotient spaces $\mathcal{Q}^{m}$. Section 1 gives a preliminary view of the equations which can be obtained by iterating and combining the reduction processes in the simplest case $m=1, n=2$ corresponding to the usual KP theory. In Section 5 we exhibit the explicit structure of the equations $\mathrm{CS}_{n}$ and $\mathrm{KP}^{(m)}$. Section 6 is devoted to the $\mathrm{KdV}_{n}^{m}$ equations. In particular we work out the case $\mathrm{KdV}_{3}^{2}$. Finally, in Section 7 we give the bihamiltonian interpretation of these equations along the lines explained in Section 2, and we make an explicit comparison with the approach based on the generalized DS procedure. 


\section{The Central System}

The present approach to fractional KdV hierarchies comes from the bihamiltonian theory of $\mathrm{KdV}$ equation. It may be suitable, therefore, to collect in this section the main ideas of this theory.

The starting point is the relation between bihamiltonian manifolds and integrable systems clarified by a theorem of Gel'fand and Zakharevich [19]. Let the phase space $\mathcal{M}$ be a $(2 n+1)$-dimensional manifold, endowed with a pencil of compatible Poisson brackets

$$
\{f, g\}_{\lambda}=\{f, g\}_{1}-\lambda\{f, g\}_{0} .
$$

We assume that this pencil has maximal rank everywhere on $\mathcal{M}$, and that the symplectic leaves of $\{f, g\}_{\lambda}$ are submanifolds of dimension $2 n$ in $\mathcal{M}$. As it was noticed by Gel'fand and Zakharevich, its Casimir function is a polynomial of degree $n$ in the parameter $\lambda$

$$
H(\lambda)=H_{0}+{ }_{1} \lambda+\ldots+H_{n} \lambda^{n},
$$

and its coefficients $\left(H_{0}, H_{1}, \ldots, H_{n}\right)$ are in involution with respect to all the Poisson brackets of the pencil. Therefore, they verify the conservation laws

$$
\frac{\partial}{\partial t_{j}} H_{k}=0
$$

where the derivative of the function $H_{k}$ is taken along the Hamiltonian vector field associated with the function $H_{j}$. These vector fields define an integrable system on $\mathcal{M}$.

This bihamiltonian strategy may be formally extended to partial differential equations in one space variable $x$, with two significant differences. The Casimir functions $H(\lambda)$ now becomes a Laurent series in $\lambda$ (or in some power of $\lambda$ ) and

Figure 2: The Full Reduction Schemes

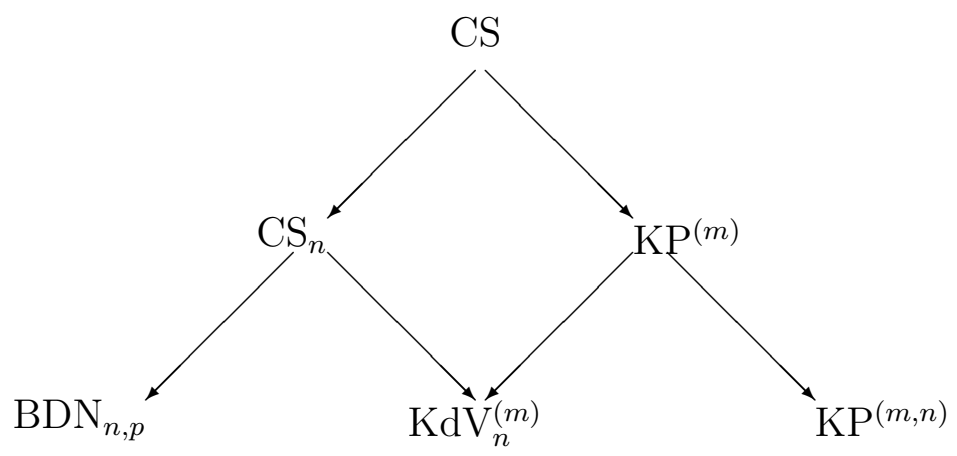


the involution relations $\left\{H_{i}, H_{j}\right\}_{\lambda}=0, i, j=0, \ldots n$, are replaced by local conservations laws (or continuity equations) of the form

$$
\frac{\partial h}{\partial t_{j}}=\partial_{x} H^{(j)}
$$

This fact is responsible for the appearance of a further important object of the theory: the current densities $H^{(j)}$. They define a new geometrical structure associated with the points of the phase space, which evolves in time along the orbits of the equations (2.4). The central system is the system of equations describing the time evolution of the current densities $H^{(j)}$.

Let us describe these features in the example of the $\mathrm{KdV}$ equations. From the bihamiltonian point of view, the KdV theory may be seen as the study of the Casimir function and the current densities associated with the Poisson pencil

$$
\dot{u}=-\frac{1}{2} v_{x x x}+2(u+\lambda) v_{x}+u_{x} v .
$$

This pencil can be obtained by a Marsden-Ratiu reduction of a Lie-Poisson pencil defined over the $\mathfrak{s l}(2)$ loop algebra [20, 21]. Here, $u$ is a point of the phase space of the KdV theory, $v$ is a covector, and the Poisson pencil is a map from the cotangent space to the tangent space.

The computation of the Casimir function is comparatively easy. Let us set $\lambda=z^{2}$, and let us introduce the Hamiltonian density $h(z)$ of the Casimir function according to

$$
H(z)=2 z \int_{S^{1}} h(z) d x .
$$

One can prove that $h(z)$ is the unique monic Laurent series

$$
h(z)=z+\sum_{l \geq 1} h_{l}(x) z^{-l}
$$

which solves the Riccati equation

$$
h_{x}+h^{2}=u+z^{2}
$$

Thus, the role of this equation is to define the Casimir function at any point $u$ of the phase space.

The computation of the current $H^{(j)}$ is, instead, a little more tricky. We have to consider the Faà di Bruno polynomials $h^{(k)}$ defined by the recursive formula

$$
h^{(k+1)}=h_{x}^{(k)}+h h^{(k)},
$$

starting from $h^{(0)}=1$. Then we have to linearly combine these polynomials, with coefficients depending on the coefficients $h_{i}$ of $h(z)$, but independent of $z$,

$$
H^{(j)}=\sum_{k=0}^{j} c_{k}^{j}\left(h_{1}, h_{2}, \ldots\right) h^{(k)}(z)
$$


in such a way to obtain Laurent series $H^{(j)}$ of the form

$$
H^{(i)}(z)=z^{j}+\sum_{l \geq 1} H_{l}^{i} z^{-l}
$$

They are the current densities we are looking for. Indeed one can prove [22, 21] that, when $u$ evolves according to the KdV hierarchy, the solution $h(z)$ of the Riccati equation (2.8) evolves exactly according to the conservation laws (2.4), with the currents (2.11). Furthermore, the current densities presently defined evolve in time according to the central system (1.2).

To arrive at the general form of the central system (1.2), we have to remark that the assumption that $h(z)$ be a solution of the Riccati equation is actually inessential. Indeed, equations (2.10) and (2.11) define the current densities $H^{(j)}$ associated with any Laurent series $h(z)$ of the form (2.7). Therefore, we can regard equations (2.4) as a system of local conservation laws for any monic Laurent series $h(z)$, whether or not it satisfies the Riccati equation (2.8). This enlarged hierarchy is the KP theory. Indeed, equations (2.4), considered on an arbitrary monic Laurent series $h(z)$, are (a possible form of) the celebrated $\mathrm{KP}$ equations [22, 15, 23].

The second remark is that also the definition (2.10) of the current densities we have used to construct the KP equations, is inessential. The central system is actually independent of the definition (2.10) of the currents $H^{(j)}$, and only rests on the particular form of their Laurent expansion (2.11). Therefore, we can eliminate any reference to a "space variable $x$ " and to a "Hamiltonian density $h(z)$ ", and we can regard the central system as defining an independent family of vector fields on the space $\mathcal{H}$ of collections of such currents. From this perspective, equations (2.10) (associated with the KP equations) and the Riccati equation (2.8) (leading to the KdV theory) are simply a set of constraints, which are compatible with the central system. Other constraints are possible as well, leading to different systems of equations. Among them there are the fractional KdV systems.

\section{The Central System and its Reductions}

In this section we begin to study the central systems and its reductions. The starting point is the observation that the CS vector fields pairwise commute. To prove this property, we begin with some remarks on its structure.

Let us consider the space $\mathcal{L}$ of truncated Laurent series

$$
l(z)=\sum_{-\infty}^{N(l)} l_{j} z^{j} .
$$

In this space the currents $H^{(j)}, j \geq 0$, introduced in Section 1 as

$$
H^{(0)}=1, \quad H^{(k)}=z^{k}+\sum_{l \geq 1} H_{l}^{k} z^{-l},
$$


determine a subspace

$$
\mathcal{H}_{+}:=\left\langle H^{(0)}, H^{(1)}, \ldots\right\rangle,
$$

transversal to the subspace

$$
\mathcal{H}_{-}:=\left\langle z^{-1}, z^{-2}, \ldots\right\rangle
$$

of the Laurent series of strictly negative degree. We can now regard any solution of CS as a subspace $\mathcal{H}_{+}(t)$ moving in $\mathcal{L}$. The characteristic property of the solutions of CS is the invariance of $\mathcal{H}_{+}(t)$ with respect to the action of the differential operators $\frac{\partial}{\partial t_{j}}+H^{(j)}$ associated with the currents.

Proposition 3.1 Along the flows of the central system the subspace $\mathcal{H}_{+}(t)$ satisfies the invariance relation

$$
\left(\frac{\partial}{\partial t_{j}}+H^{(j)}\right)\left(\mathcal{H}_{+}\right) \subset \mathcal{H}_{+} .
$$

Proof. We show that this relation completely defines CS. Since the currents $H^{(k)}$ form a basis of $\mathcal{H}_{+}$, the invariance property (3.5) entails the existence of coefficients $c_{l}^{j k}$ independent of $z$ such that

$$
\frac{\partial H^{(k)}}{\partial t_{j}}+H^{(j)} H^{(k)}=\sum_{l=0}^{j+k} c_{l}^{j k} H^{(l)} .
$$

These coefficients are easily determined by comparing the expansion in powers of $z$ of both sides of equation (3.6). The final result is equation (1.2) defining the central system.

As an immediate consequence of the previous result, we obtain the following compact representation of CS. We denote by $\pi_{+}$and $\pi_{-}$the canonical projections associated with the decomposition

$$
\mathcal{L}=\mathcal{H}_{+} \oplus \mathcal{H}_{-}
$$

of the space $\mathcal{L}$. Then we have

Proposition 3.2 The central system is the family of dynamical systems on the currents $H^{(j)}$ given by

$$
\frac{\partial}{\partial t_{k}} H^{(j)}=-\pi_{-}\left(H^{(j)} H^{(k)}\right) .
$$


Proof. We apply $\pi_{-}$to both sides of (3.6) and we get

$$
\pi_{-}\left(\frac{\partial H^{(k)}}{\partial t_{j}}+H^{(j)} H^{(k)}\right)=0 .
$$

The assertion follows from the fact that $H^{(k)}=z^{k}+\sum_{l \geq 1} H_{l}^{k} z^{-l}$ so that $\left(\frac{\partial H^{(k)}}{\partial t_{j}}\right) \in \mathcal{H}_{-}$.

From the symmetry in $j$ and $k$ of the right-hand side of (3.8), we finally obtain the following

Proposition 3.3 Every solution of CS satisfies the exactness condition

$$
\frac{\partial}{\partial t_{k}} H^{(j)}=\frac{\partial}{\partial t_{j}} H^{(k)}
$$

We are now ready to prove the commutativity property of the CS flows.

Proposition 3.4 The flows of the central system pairwise commute.

Proof. We compute the action of the commutator $\left[X_{j}, X_{k}\right]$ of two vector fields of the hierarchy on a generic current:

$$
\left[X_{j}, X_{k}\right]\left(H^{(i)}\right)=\frac{\partial}{\partial t_{j}} \frac{\partial H^{(i)}}{\partial t_{k}}-\frac{\partial}{\partial t_{k}} \frac{\partial H^{(i)}}{\partial t_{j}} .
$$

We remark that that this quantity belongs to $\mathcal{H}_{-}$, thanks to the specific form of $H^{(j)}$. Then we observe that, using the exactness property (3.9), this commutator can be also written in the form

$$
\left[X_{j}, X_{k}\right]\left(H^{(i)}\right)=\left[\frac{\partial}{\partial t_{j}}+H^{(j)}, \frac{\partial}{\partial t_{k}}+H^{(k)}\right] H^{(i)},
$$

so that the commutator of the vector fields coincides with the commutator of the operators $\frac{\partial}{\partial t_{j}}+H^{(j)}$ associated with the currents. Hence the invariance property (3.5) entails that $\left[X_{j}, X_{k}\right]\left(H^{(i)}\right)$ belongs to the subspace $\mathcal{H}_{+}$for all the $H^{(i)}$ 's. But $\mathcal{H}_{+} \cap \mathcal{H}_{-}=\{0\}$, and therefore $\left[X_{j}, X_{k}\right]$ vanishes. 


\subsection{The Invariant Submanifolds $\mathcal{S}_{n}$}

From the commutativity of the flows it follows that the set $\mathcal{Z}_{n}$ of zeroes of the vector field $X_{n}$, defined by the quadratic equations

$$
H^{(k+n)}-H^{(k)} H^{(n)}+\sum_{l=1}^{k} H_{l}^{n} H^{(k-l)}+\sum_{l=1}^{n} H_{l}^{k} H^{(n-l)}=0,
$$

is an invariant submanifold for CS. Moreover, on $\mathcal{Z}_{n}$ we have

$$
\frac{\partial H^{(n)}}{\partial t_{j}}=\frac{\partial H^{(j)}}{\partial t_{n}}=0
$$

thanks to the exactness property (3.9). Therefore the manifold $\mathcal{Z}_{n}$ is foliated by invariant submanifolds defined by the equation $H^{(n)}=$ constant. Among all these leaves we choose the one given by the condition

$$
H^{(n)}=z^{n}
$$

which is the counterpart of the choice usually considered in the reduction theory from KP to the n-GD hierarchies [13, 14.

Definition 3.5 We call $\mathcal{S}_{n}$ the submanifold of the zeroes of $X_{n}$, where the current $H^{(n)}$ satisfies the relation (3.13).

The equations defining this submanifold can be explicitly found by eliminating $H^{(n)}$ from $(3.11)$ by means of the constraints (3.13). This leads to the conclusion that $\mathcal{S}_{n}$ is the subset of $\mathcal{H}$ defined by the equations

$$
H^{(j+n)}=z^{n} H^{(j)}-\sum_{l=1}^{n} H_{l}^{j} H^{(n-l)} .
$$

Therefore, it is parametrized by the first $n-1$ currents $\left(H^{(1)}, \ldots, H^{(n-1)}\right)$. A more intrinsic characterization is provided by the following

Proposition 3.6 The submanifold $\mathcal{S}_{n}$ is the subset of $\mathcal{H}$ given by the equation

$$
z^{n}\left(\mathcal{H}_{+}\right) \subset \mathcal{H}_{+}
$$

i.e., the set of the points where the operator of multiplication by $z^{n}$ leaves the space $\mathcal{H}_{+}$invariant.

Proof. First of all, it is easily shown that the condition (3.15) is necessary. It is enough, indeed, to observe that the invariance relation

$$
\left(\frac{\partial}{\partial t_{n}}+H^{(n)}\right)\left(\mathcal{H}_{+}\right) \subset \mathcal{H}_{+}
$$


characterizing the vector field $X_{n}$, at the points of $\mathcal{S}_{n}$ boils down to the relation (3.15) thanks to 3.13$)$.

To show that the condition is also sufficient, let us remark that $z^{n} \cdot H^{(0)}=$ $z^{n} \cdot 1=z^{n}$ belongs to $\mathcal{H}_{+}$and then can be developed as

$$
z^{n}=\sum_{l=0}^{n} c_{l}^{n} H^{(l)}
$$

By a comparison between the coefficients of the positive powers of $z$ we obtain that

$$
z^{n}=H^{(n)} .
$$

In the same way it can be proved that

$$
z^{n} H^{(j)}=H^{(j+n)}+\sum_{l=1}^{n} H_{l}^{j} H^{(n-l)} .
$$

Now it is enough to write this relation in the form

$$
H^{(j+n)}-z^{n} H^{(j)}+\sum_{l=1}^{n} H_{l}^{j} H^{(n-l)}=0
$$

with the condition (3.14).

\subsection{The Quotient Spaces $\mathcal{Q}_{m}$}

Let us consider now the second process of reduction. We concentrate on a vector field of the hierarchy, say $X_{m}$, and we denote the corresponding time $t_{m}$ by

$$
t_{m}=x
$$

in order to point out its special role in the reduction. This amounts to convert $t_{m}$ into a "space variable", in the terminology used in the theory of KP equations. It is worthwhile to remark that the projection to $\mathcal{Q}_{m}$ with $m \neq 1$ formalizes the procedure that in the physics literature [25] is usually described as the "exchange $x \leftrightarrow t_{m}$ ".

Definition 3.7 We call $\mathcal{Q}_{m}$ the space of the solutions of the $m$-th flow of the central system, i.e., the space of orbits of the vector field $X_{m}$.

Thanks to the commutativity condition, any vector field $X_{j}$ of CS induces a flow, which we still denote by $X_{j}$, on $\mathcal{Q}_{m}$. The first problem to be solved is to characterize the variety $\mathcal{Q}_{m}$. 
Proposition 3.8 The quotient spaces $\mathcal{Q}_{m}$ can be identified with the space of $m$-tuples $\left\{H^{(a)}(z)\right\}_{a=1, \ldots, m}$ of Laurent series of the form

$$
H^{(a)}(z)=z^{a}+\sum_{l \geq 1} H_{l}^{a}(x) z^{-l},
$$

whose coefficients are functions of the space variable $x$.

Proof. It suffices to remark that the equations defining the vector field $X_{m}$ may be written in the form of recursion relations

$$
H^{(j+m)}=\left(\frac{\partial}{\partial x}+H^{(m)}\right) H^{(j)}-\sum_{l=1}^{j} H_{l}^{m} H^{(j-l)}-\sum_{l=1}^{m} H_{l}^{j} H^{(m-l)} .
$$

They allow to compute the currents $\left(H^{(m+1)}, H^{(m+2)}, \ldots\right)$ as differential polynomials in the first $m$ currents $\left(H^{(1)}, \ldots, H^{(m)}\right)$. Explicitly, this means that the coefficients of $\left(H^{(m+1)}, H^{(m+2)}, \ldots\right)$ are polynomials in the coefficients of $\left(H^{(1)}, \ldots, H^{(m)}\right)$ and their $x$-derivatives. Since the generators $\left(H^{(1)}, \ldots, H^{(m)}\right)$ depend on $x$ in a completely arbitrary way, we conclude that the space of orbits of the vector field $X_{m}$ coincides with the space of $m$-tuples of Laurent series (3.22).

\section{Preliminary examples of reduced equations}

Let us consider the simplest examples of reductions of the central system. We first consider the submanifold $\mathcal{S}_{2}$. According to equation (3.14), it is parametrized by the single Laurent series $H^{(1)}$. To simplify the notations we set

$$
h(z):=H^{(1)}=z+\sum_{l \geq 1} h_{l} z^{-l} .
$$

The constraints defining $\mathcal{S}_{2}$ are

$$
\begin{aligned}
H^{(2)} & =z^{2}, \quad H^{(3)}=z^{2} h(z)-h_{1} h(z)-h_{2}, \quad H^{(4)}=z^{4} \\
H^{(5)} & =z^{2} H^{(3)}-H_{1}^{3} h(z)-H_{2}^{3} \\
& =\left(z^{4}-h_{1} z^{2}+h_{1}^{2}-h_{3}\right) h(z)-h_{2} z^{2}+h_{2} h_{1}-h_{4},
\end{aligned}
$$

and so on. To construct $\mathrm{CS}_{2}$, it is sufficient to plug these constraints in the equation

$$
\frac{\partial h(z)}{\partial t_{j}}=H^{(j+1)}-h H^{(j)}+\sum_{l=1}^{j} h_{l} H^{(j-l)}+H_{1}^{j} .
$$


Table 1: The first $\mathrm{CS}_{2}$ equations

$$
\begin{array}{ll}
\frac{\partial h_{1}}{\partial t_{1}}=-2 h_{2} & \frac{\partial h_{1}}{\partial t_{3}}=-2 h_{4}+2 h_{1} h_{2} \\
\frac{\partial h_{2}}{\partial t_{1}}=-\left(2 h_{3}+h_{1}^{2}\right) & \frac{\partial h_{2}}{\partial t_{3}}=-2 h_{5}+h_{2}^{2}+h_{1}^{3} \\
\frac{\partial h_{3}}{\partial t_{1}}=-\left(2 h_{4}+2 h_{1} h_{2}\right) & \frac{\partial h_{3}}{\partial t_{3}}=-2 h_{6}+2 h_{1}^{2} h_{2} \\
\frac{\partial h_{4}}{\partial t_{1}}=-\left(2 h_{5}+2 h_{1} h_{3}+h_{2}^{2}\right) & \frac{\partial h_{4}}{\partial t_{3}}=-2 h_{7}+2 h_{1}^{2} h_{3}-h_{3}^{2}+h_{1} h_{2}^{2} \\
\frac{\partial h_{5}}{\partial t_{1}}=-\left(2 h_{6}+2 h_{1} h_{4}+2 h_{2} h_{3}\right) & \frac{\partial h_{5}}{\partial t_{3}}=-2 h_{8}-2 h_{3} h_{4}+2 h_{1}^{2} h_{4}+2 h_{2} h_{2} h_{3} \\
\frac{\partial h_{1}}{\partial t_{5}}=2 h_{3} h_{2}-2 h_{6}+2 h_{1} h_{4}-2\left(h_{1}\right)^{2} h_{2} \\
\frac{\partial h_{2}}{\partial t_{5}}=h_{3}^{2}+2 h_{2} h_{4}+h_{1}^{2} h_{3}-2 h_{7}-h_{1} h_{2}^{2}-\left(h_{1}\right)^{4} \\
\frac{\partial h_{3}}{\partial t_{5}}=2 h_{3} h_{4}-2 h_{1}^{3} h_{2}-2 h_{8}+2 h_{1} h_{3} h_{2} \\
\frac{\partial h_{4}}{\partial t_{5}}=-2 h_{9}+3 h_{1} h_{3}^{2}-2 h_{1}^{3} h_{3}+h_{4}^{2}-h_{2}^{2} h_{1}^{2}+h_{2}^{2} h_{3} \\
\frac{\partial h_{5}}{\partial t_{5}}=2 h_{2} h_{3}^{2}-2 h_{2} h_{1}^{2} h_{3}-2 h_{10}+4 h_{1} h_{3} h_{4}-2 h_{1}^{3} h_{4}
\end{array}
$$

to get the equations displayed in Table I. It is worthwhile to display also the first $\mathrm{CS}_{3}$ equations. By setting

$$
k(z):=H^{(2)}=z^{2}+\sum_{l \geq 1} k_{l} z^{-l}
$$

along with (4.1) and using the first parametric equations of the submanifold $\mathrm{S}_{3}$

$$
\begin{aligned}
& H^{(3)}=z^{3} \\
& H^{(4)}=z^{3} h-h_{1} k-h_{2} h-h_{3} \\
& H^{(5)}=z^{3} k-k_{1} k-k_{2} h-k_{3}
\end{aligned}
$$

we get the equations collected in Table II.

Let us now consider the quotient space $\mathcal{Q}_{1}$. We keep the usual notation $H^{(1)}=h$, but we recall that here $h$ must be considered as a a Laurent series $h=z+\sum_{l \geq 1} h_{l}(x) / z^{l}$, whose coefficients depend on the space variable $x$. By 
Table 2: The first $\mathrm{CS}_{3}$ equations

$$
\begin{gathered}
\frac{\partial h_{1}}{\partial t_{1}}=k_{1}-2 h_{2} \\
\frac{\partial h_{2}}{\partial t_{1}}=k_{2}-2 h_{3}-h_{1}^{2} \\
\frac{\partial h_{3}}{\partial t_{1}}=k_{3}-2 h_{2} h_{1}-2 h_{4} \\
\frac{\partial h_{4}}{\partial t_{1}}=k_{4}-2 h_{1} h_{3}-2 h_{5}-h_{2}^{2} \\
\frac{\partial h_{1}}{\partial t_{2}}=\frac{\partial k_{1}}{\partial t_{1}}=-h_{3}-k_{2}+h_{1}^{2} \\
\frac{\partial h_{2}}{\partial t_{2}}=\frac{\partial k_{2}}{\partial t_{1}}=-h_{1} k_{1}-h_{4}-k_{3}+h_{2} h_{1} \\
\frac{\partial h_{3}}{\partial t_{2}}=\frac{\partial k_{3}}{\partial t_{1}}=-k_{1} h_{2}-h_{1} k_{2}-h_{5}-k_{4}+h_{1} h_{3} \\
\frac{\partial h_{4}}{\partial t_{2}}=\frac{\partial k_{4}}{\partial t_{1}}=-k_{1} h_{3}-k_{2} h_{2}-h_{1} k_{3}-h_{6}-k_{5}+h_{1} h_{4} \\
\frac{\partial k_{1}}{\partial t_{2}}=h_{4}+h_{1} k_{1}-h_{2} h_{1}-2 k_{3} \\
\frac{\partial k_{2}}{\partial t_{2}}=h_{5}-h_{2}^{2}-h_{1} k_{2}+2 k_{1} h_{2}-2 k_{4}-k_{1}^{2} \\
\frac{\partial k_{3}}{\partial t_{2}}=h_{6}-h_{1} k_{3}-h_{2} h_{3}-2 k_{1} k_{2}+2 k_{1} h_{3}-2 k_{5} \\
\frac{\partial k_{4}}{\partial t_{2}}=h_{7}-h_{1} k_{4}-h_{2} h_{4}-2 k_{1} k_{3}+2 h_{4} k_{1}-2 k_{6}-k_{2}^{2}
\end{gathered}
$$

using the equations of the vector field $X_{1}$, from (3.22) we obtain the relations

$$
\begin{aligned}
H^{(2)} & =h_{x}+h^{2}-2 h_{1} \\
H^{(3)} & =H_{x}^{(2)}+h H^{(2)}-h_{1} h-h_{2} \\
& =h_{x x}+3 h h_{x}+h^{3}-3 h_{1} h-3\left(h_{1 x}+h_{2}\right)
\end{aligned}
$$

The $\mathrm{KP}^{(1)}$ equations, describing the projection of $\mathrm{CS}$ on $\mathcal{Q}_{1}$, are obtained by introducing the recursion relation (4.6) in the equation

$$
\frac{\partial h}{\partial t_{j}}=H^{(j+1)}-h H^{(j)}+\sum_{l=1}^{j} h_{l} H^{(j-l)}+H_{1}^{j}=\frac{\partial H^{(j)}}{\partial x} .
$$

Expanding these equations in powers of $z$, one constructs the equations displayed in Table III. After a suitable coordinate change [15, 22, 23], these equa- 
Table 3: The first $\mathrm{KP}^{(1)}$ equations

$$
\begin{array}{ll}
\frac{\partial h_{1}}{\partial t_{1}}=h_{1 x} & \frac{\partial h_{1}}{\partial t_{2}}=\partial_{x}\left(h_{1 x}+2 h_{2}\right) \\
\frac{\partial h_{2}}{\partial t_{1}}=h_{2 x} & \frac{\partial h_{2}}{\partial t_{2}}=\partial_{x}\left(h_{2 x}+h_{1}^{2}+2 h_{3}\right) \\
\frac{\partial h_{3}}{\partial t_{1}}=h_{3 x} & \frac{\partial h_{3}}{\partial t_{2}}=\partial_{x}\left(h_{3 x}+2 h_{4}+2 h_{1} h_{2}\right) \\
\frac{\partial h_{1}}{\partial t_{3}}=\partial_{x}\left(h_{1 x x}+3 h_{2 x}+3 h_{3}\right) & \\
\frac{\partial h_{2}}{\partial t_{3}}=\partial_{x}\left(h_{2 x x}+3 h_{3 x}+3 h_{1} h_{1 x}+3 h_{4}+3 h_{1} h_{2}\right) & \\
\frac{\partial h_{3}}{\partial t_{3}}=\partial_{x}\left(h_{3 x x}+3 h_{4 x}+3 h_{1} h_{2 x}+3 h_{1 x} h_{2}\right. & \\
& \left.+3 h_{1} h_{3}+3 h_{2}^{2}+h_{1}^{3}\right)
\end{array}
$$

tions coincide with the usual KP equations in the $1+1$ formalism.

We shall now consider the double reduction on the space $\mathcal{Q}_{2}^{1}$. We can start either from $\mathrm{KP}^{(1)}$ or from $\mathrm{CS}_{2}$. In the first case, we have only to impose the constraint

$$
H^{(2)}=h_{x}+h^{2}-2 h_{1}=z^{2}
$$

that is,

$$
\begin{aligned}
& 2 h_{2}+h_{1 x}=0 \\
& 2 h_{3}+h_{2 x}+h_{1}^{2}=0 \\
& 2 h_{4}+h_{3 x}+2 h_{1} h_{2}=0
\end{aligned}
$$

on the currents (4.6). As it is well-known [22], this is the constraint defining the Hamiltonians of the KdV hierarchy, which leads from the KP equations to the KdV equations

In the second case, we can proceed in three steps. First, we use the exactness condition (3.9) to write the equations of Table I in the form of Table IV. Then we transform the time $t_{1}$ in a space variable $x$, and we regard the first $\mathrm{CS}_{2}$ 
Table 4: The $C S_{2}$ equations as conservation laws.

$$
\begin{array}{rlrl}
\frac{\partial h_{1}}{\partial t_{1}}=-2 h_{2} & \frac{\partial h_{1}}{\partial t_{3}}=\frac{\partial}{\partial t_{1}}\left(h_{3}-h_{1}^{2}\right) \\
\frac{\partial h_{2}}{\partial t_{1}}=-\left(2 h_{3}+h_{1}^{2}\right) & \frac{\partial h_{2}}{\partial t_{3}}=\frac{\partial}{\partial t_{1}}\left(h_{4}-h_{1} h_{2}\right) \\
\frac{\partial h_{3}}{\partial t_{1}}=-\left(2 h_{4}+2 h_{1} h_{2}\right) & \frac{\partial h_{3}}{\partial t_{3}}=\frac{\partial}{\partial t_{1}}\left(h_{5}-h_{1} h_{3}\right) \\
\frac{\partial h_{4}}{\partial t_{1}}=-\left(2 h_{5}+2 h_{1} h_{3}+h_{2}^{2}\right) & \frac{\partial h_{4}}{\partial t_{3}}=\frac{\partial}{\partial t_{1}}\left(h_{6}-h_{1} h_{4}\right) \\
\frac{\partial h_{5}}{\partial t_{1}}=-\left(2 h_{6}+2 h_{1} h_{4}+2 h_{2} h_{3}\right) & \frac{\partial h_{5}}{\partial t_{3}}=\frac{\partial}{\partial t_{1}}\left(h_{7}-h_{1} h_{5}\right) \\
\frac{\partial h_{1}}{\partial t_{5}}=\frac{\partial}{\partial t_{1}}\left(h_{5}-2 h_{1} h_{3}+h_{1}^{3}\right) & \\
\frac{\partial h_{2}}{\partial t_{5}}=\frac{\partial}{\partial t_{1}}\left(h_{6}-h_{2} h_{3}-h_{1} h_{4}+h_{2} h_{1}^{2}\right) \\
\frac{\partial h_{3}}{\partial t_{5}}=\frac{\partial}{\partial t_{1}}\left(h_{7}-h_{3}^{2}-h_{1} h_{5}+h_{3} h_{1}^{2}\right) \\
\frac{\partial h_{4}}{\partial t_{5}}=\frac{\partial}{\partial t_{1}}\left(-h_{3} h_{4}+h_{1}^{2} h_{4}-h_{1} h_{6}+h_{8}\right) \\
\frac{\partial h_{5}}{\partial t_{5}}=\frac{\partial}{\partial t_{1}}\left(-h_{3} h_{5}-h_{1} h_{7}+h_{9}+h_{1}^{2} h_{5}\right)
\end{array}
$$

equation as a set of recursive relations

$$
\begin{aligned}
& 2 h_{2}=-h_{1 x} \\
& 2 h_{3}=-\left(h_{2 x}+h_{1}^{2}\right) \\
& 2 h_{4}=-\left(h_{3 x}+2 h_{1} h_{2}\right)
\end{aligned}
$$

They allow to identify the space $\mathcal{Q}_{2}^{1}$ with the space of scalar functions $u \equiv 2 h_{1}$ in one space variable $x$ (the phase space of $\mathrm{KdV}$ ). Finally, we insert these constraints in the first component of each vector field $X_{j}$ of $\mathrm{CS}_{2}$ (the other components can be neglected, since they give rise only to differential consequences of the previous ones). Once again, we get the KdV equations

$$
\begin{aligned}
& \frac{\partial u}{\partial t_{3}}=\partial_{x}\left(h_{3}-h_{1}^{2}\right)=\frac{1}{8} \partial_{x}\left(u_{x x}-3 u^{2}\right) \\
& \frac{\partial u}{\partial t_{5}}=\partial_{x}\left(h_{5}-2 h_{1} h_{3}+h_{1}^{3}\right)=\frac{1}{32} \partial_{x}\left(u_{x x x x}-10 u u_{x x}-5 u_{x}^{2}+10 u^{3}\right)
\end{aligned}
$$

and so on. This computation clearly points out how the projection process allows to transform a family of dynamical system into a hierarchy of evolution 
partial differential equation in $1+1$ dimensions (one space-dimension and one time-dimension).

We end this section by briefly describing another example of double reduction of CS, on the intersection $\mathcal{S}_{n} \cap \mathcal{Z}_{p}$. In this case we obtain systems of ordinary differential equations in a finite number of fields. Let us exemplify this feature by considering the intersection $\mathcal{S}_{2} \cap \mathcal{Z}_{5}$. Looking at the equations defining $X_{5}$ in Table III, we obtain the constraints

$$
\begin{aligned}
& h_{6}=h_{1} h_{4}+h_{2} h_{3}-h_{1}^{2} h_{2} \\
& h_{7}=h_{2} h_{4}+\frac{1}{2}\left(h_{3}^{2}+h_{1}^{2} h_{3}-h_{1} h_{2}^{2}-h_{1}^{4}\right) \\
& h_{8}=h_{3} h_{4}+h_{1} h_{2} h_{3}-h_{1}^{3} h_{2}
\end{aligned}
$$

which are an infinite system of recurrence relations allowing to express all the $h_{l}$ 's, for $l \geq 6$, as polynomial functions of $h_{1}, \ldots, h_{5}$. Therefore the invariant submanifold $\mathcal{S}_{2} \cap \mathcal{Z}_{5}$ is 5 -dimensional . The restriction of $\mathrm{CS}_{2}$ to this submanifold is simply constructed by plugging the constraints (4.12) in the first five components of the vector fields $X_{1}$ and $X_{3}$. We get the equations

$$
\begin{array}{ll}
\frac{\partial h_{1}}{\partial t_{1}}=-2 h_{2} & \frac{\partial h_{1}}{\partial t_{3}}=-\left(2 h_{4}-2 h_{1} h_{2}\right) \\
\frac{\partial h_{2}}{\partial t_{1}}=-\left(2 h_{3}+h_{1}^{2}\right) & \frac{\partial h_{2}}{\partial t_{3}}=-\left(2 h_{5}-h_{2}^{2}-h_{1}^{3}\right) \\
\frac{\partial h_{3}}{\partial t_{1}}=-\left(2 h_{4}+2 h_{1} h_{2}\right) & \frac{\partial h_{3}}{\partial t_{3}}=-\left(2 h_{1} h_{4}+2 h_{2} h_{3}-4 h_{1}^{2} h_{2}\right) \\
\frac{\partial h_{4}}{\partial t_{1}}=-\left(2 h_{5}+2 h_{1} h_{3}+h_{2}^{2}\right) & \frac{\partial h_{4}}{\partial t_{3}}=-\left(2 h_{3}^{2}-h_{1}^{2} h_{3}-h_{1}^{4}-2 h_{1} h_{2}^{2}+2 h_{2} h_{4}\right) \\
\frac{\partial h_{5}}{\partial t_{1}}=-\left(4 h_{1} h_{4}+4 h_{2} h_{3}-2 h_{1}^{2} h_{2}\right) & \frac{\partial h_{5}}{\partial t_{3}}=-\left(4 h_{3} h_{4}-2 h_{1}^{3} h_{2}-2 h_{1}^{2} h_{4}\right)
\end{array}
$$

The equations corresponding to the vector fields $X_{2 j+1}$, for $j \geq 2$, are combinations of equations (4.13). Hence the equations we have written represent the whole reduced system. After a suitable coordinates change, they coincide with the second Novikov system [17] relative to the KdV hierarchy.

\section{The equations $\mathrm{CS}_{n}$ and $\mathrm{KP}^{(m)}$}

In Section 3 we explained the geometric process leading to the $\mathrm{CS}_{n}$ and $\mathrm{KP}^{(m)}$ equations. In this section we shall give an algebraic definition of these equations by using the concept of Fa⿳亠 di Bruno basis associated with a (finite set of) monic Laurent series. We present this idea first for the $\mathrm{CS}_{n}$ case. In Section 3 we showed that this is a system of ordinary differential equations in the 
first $(n-1)$ currents

$$
H^{(a)}=z^{a}+\sum_{l \geq 1} H_{l}^{a} z^{-l}
$$

for $a=1, \ldots, n-1$. As usual, we set $H^{(0)}=1$ and we we consider the point $H^{(n)}=z^{n}$ in the space $\mathcal{L}$ of all truncated Laurent series. We call stationary Fà̀ di Bruno basis at the point $z^{n}$ the basis of $\mathcal{L}$ defined by the iterates

$$
\begin{aligned}
& F^{(j+n)}=z^{n} \cdot F^{(j)} \\
& F^{(a)}=H^{(a)} \quad \text { for } a=0, \ldots, n-1,
\end{aligned}
$$

of the initial generators $\left(H^{(0)}, \ldots, H^{(n-1)}\right)$, for $j \in \mathbb{Z}$. Let us denote with $\mathcal{H}_{+}$ the subspace of $\mathcal{L}$ spanned by the nonnegative elements of the Faà di Bruno basis at the point $z^{n}$, and with $\mathcal{H}_{-}$the subspace spanned by the negative ones. We decompose $\mathcal{L}$ in the direct sum

$$
\mathcal{L}=\mathcal{H}_{+} \oplus \mathcal{H}_{-}
$$

and we call

$$
H^{(j)}=\pi_{+}\left(z^{j}\right)
$$

the projections on $\mathcal{H}_{+}$of the powers $z^{j}$ with respect to this decomposition.

Definition 5.1 (Second definition of $\mathbf{C S}_{n}$ ) We call $C S_{n}$ the dynamical system in the currents $\left(H^{(1)}, \ldots, H^{(n-1)}\right)$ defined by

$$
\frac{\partial H^{(a)}}{\partial t_{j}}=-\pi_{-}\left(H^{(a)} H^{(j)}\right),
$$

for $a=1, \ldots, n-1$, and $j \in \mathbb{N}$.

We proceed in the same way for the $\mathrm{KP}^{(m)}$ equations. In this case $\mathcal{L}$ is the space of truncated Laurent series whose coefficients are functions of the space variable $x$, and we consider the point

$$
H^{(m)}=z^{m}+\sum_{l \geq 1} H_{l}^{m} z^{-l}
$$

We call differential Faà di Bruno basis at the point $H^{(m)}$ the basis of $\mathcal{L}$ defined by the iterates

$$
\begin{aligned}
& F^{(j+m)}=\left(\partial_{x}+H^{(m)}\right) \cdot F^{(j)} \\
& F^{(a)}=H^{(a)} \quad \text { for } a=0, \ldots, m-1,
\end{aligned}
$$

of the initial generators $\left(H^{(0)}, \ldots, H^{(m-1)}\right)$, for $j \in \mathbb{Z}$. We observe that the computation of the elements $H^{(j)}$, with $j<0$, of the Faà di Bruno basis does 
not require any integration, since the recurrence relation (5.7) can be inverted in a purely algebraic way. As before, we decompose the space $\mathcal{L}$ in the form (5.3), and we introduce the projections

$$
H^{(j)}=\pi_{+}\left(z^{j}\right)
$$

of the powers $z^{j}$. Even if we used the same notations to point out the analogy between the two situations, one has to keep in mind that they live in different spaces, and that the Faà di Bruno basis are defined in a different way.

Definition 5.2 (Second definition of $\mathbf{K P}^{(m)}$ ) We call $K P^{(m)}$ the system of evolutionary partial differential equations in the currents $\left(H^{(1)}, \ldots, H^{(m)}\right)$ defined by

$$
\frac{\partial H^{(a)}}{\partial t_{j}}=-\pi_{-}\left(H^{(a)} H^{(j)}\right),
$$

for $a=1, \ldots, m$, and $j \in \mathbb{N}$.

Now we have to show that the equations defined above coincide with the ones obtained by the reduction process.

Proposition 5.3 The equations (5.5) and (5.9) constructed by the Fa⿳亠 di Bruno basis algorithm coincide with the reduced equations $C S_{n}$ and $K P^{(m)}$ defined by the reduction scheme.

Proof. We call $\widehat{\mathcal{H}}_{+}$the subspace of $\mathcal{L}$ spanned by the currents $H^{(j)}$ of the central system (the same subspace that we denoted with $\mathcal{H}_{+}$in Section 3 ). We consider $\widehat{\mathcal{H}}_{+}$at a generic point of the submanifold $\mathcal{S}_{n}$. From (3.15), at the points of $\mathcal{S}_{n}$ the subspace $\widehat{\mathcal{H}}_{+}$is invariant with respect to multiplication by $z^{n}$. Hence it contains all nonnegative elements of the stationary Faà di Bruno basis associated with the point $z^{n}$. Therefore $\widehat{\mathcal{H}}_{+}$at the points of $\mathcal{S}_{n}$ coincides with the subspace $\mathcal{H}_{+}$appearing in the decomposition (5.3), constructed by choosing as generators the currents $\left(H^{(1)}, \ldots, H^{(n-1)}\right)$ defining the point of $\mathcal{S}_{n}$. It follows that the projection (5.4) belongs to $\widehat{\mathcal{H}}_{+}$, and therefore coincides with the corresponding current of CS evaluated at $\mathcal{S}_{n}$. The first part of the proposition then follows from Proposition 3.2.

As far as the $\mathrm{KP}^{(m)}$ equations are concerned, one can argue in the same way. In this case we evaluate the subspace $\widehat{\mathcal{H}}_{+}$at the points of a generic integral curve of the vector field $X_{m}$. We use the invariance property (3.5) to conclude that at these points the subspace $\widehat{\mathcal{H}}_{+}$contains all nonnegative elements of the differential Faà di Bruno basis (5.7), and therefore it coincides with the subspace $\mathcal{H}_{+}$associated with the point $H^{(m)}$. This observation leads to the conclusion that the projection (5.8) coincides with the current $H^{(j)}$ of CS evaluated at the points of the integral curve of the vector field $X_{m}$. This suffices to prove the coincidence between the $\mathrm{KP}^{(m)}$ equations obtained by projection and equations (5.9). 
To enlighten the (differential) Faà di Bruno algorithm, we consider the $\mathrm{KP}^{(2)}$ equations. We put $h=H^{(1)}$ and $k=H^{(2)}$. The first elements of the Faà di Bruno basis at the point $k$, associated with the generators $(1, h)$, are

$$
\begin{array}{ll}
F^{(0)}=1 & F^{(3)}=h_{x}+k h \\
F^{(1)}=h & F^{(4)}=k_{x}+k^{2} \\
F^{(2)}=k & F^{(5)}=h_{x x}+2 k h_{x}+h k_{x}+k^{2} h .
\end{array}
$$

We combine these elements in the form

$$
\begin{array}{ll}
H^{(1)}=F^{(1)} & H^{(3)}=F^{(3)}-h_{1} F^{(1)}-\left(h_{2}+k_{1}\right) F^{(0)} \\
H^{(2)}=F^{(2)} & H^{(4)}=F^{(4)}-2 k_{1} F^{(1)}-2 k_{2} F^{(0)}
\end{array}
$$

in order to obtain Laurent series with the asymptotic behavior $H^{(j)}=z^{j}+$ $O\left(z^{-1}\right)$. They are the currents $H^{(j)}$ written as differential polynomials of $h$ and $k$. To obtain the $\mathrm{KP}^{(2)}$ equations we can either compute the projections $\pi_{-}\left(h H^{(j)}\right)$ and $\pi_{-}\left(k H^{(j)}\right)$, or write the equations

$$
\begin{aligned}
\frac{\partial}{\partial t_{j}} h & =H^{(j+1)}-h H^{(j)}+\sum_{l=1}^{j} h_{l} H^{(j-l)}+H_{1}^{j} \\
\frac{\partial}{\partial t_{j}} k & =\partial_{x} H^{(j)} .
\end{aligned}
$$

In particular, the first vector field of the $\mathrm{KP}^{(2)}$ hierarchy is given by

$$
\begin{aligned}
& \frac{\partial}{\partial t_{1}} h=k-h^{2}+2 h_{1} \\
& \frac{\partial}{\partial t_{1}} k=\partial_{x} h .
\end{aligned}
$$

This computation allows to illustrate the link between $\mathrm{KP}^{(1)}$ and $\mathrm{KP}^{(2)}$. Indeed we write equations (5.13) in the form

$$
\begin{aligned}
& k=\frac{\partial}{\partial t_{1}} h+h^{2}-2 h_{1} \\
& \partial_{x} h=\frac{\partial}{\partial t_{1}} k
\end{aligned}
$$

and we take the $x$-derivative of the first equation above to get

$$
\partial_{x} k=\frac{\partial}{\partial t_{1}} h_{x}+2 h h_{x}-2 h_{1 x}
$$


By substitution we get

$$
\partial_{x} k=\frac{\partial^{2}}{\partial t_{1}^{2}} k+2 h \frac{\partial}{\partial t_{1}} k-2 \frac{\partial}{\partial t_{1}} k_{1} .
$$

By this process, we can express $h, k$, and the collection of their "space derivatives" $\left(h_{x}, k_{x} ; h_{x x}, k_{x x} ; \ldots\right)$ as $t_{1}$-differential polynomials in $h$. As a consequence, all the currents $H^{(j)}$ are expressed as $t_{1}$-differential polynomials in $h$, and the $\mathrm{KP}^{(2)}$-system (5.12) reduces to the $\mathrm{KP}^{(1)}$ equations with $x=t_{1}$,

$$
\frac{\partial}{\partial t_{j}} h=\frac{\partial}{\partial t_{1}} H^{(j)}
$$

Conversely, the $\mathrm{KP}^{(2)}$ hierarchy can be obtained as a differential prolongation of $\mathrm{KP}^{(1)}$. More precisely, starting from $\mathrm{KP}^{(1)}$, one has to put $k:=H^{(2)}$ and to use the definition of $k$ and the second equation of $\mathrm{KP}^{(1)}$,

$$
\begin{aligned}
& k=h_{x}+h^{2}-2 h_{1} \\
& \frac{\partial}{\partial t_{2}} h=k_{x}
\end{aligned}
$$

to express all the $x$-derivatives of $h$ in terms of $h, k$ and their $t_{2}$-derivatives. At this point one simply changes the names of the variables $x$ and $t_{2}$, by putting at first $x \mapsto t_{1}$ and then $t_{2} \mapsto x$. This kind of procedure is easily extended to describe the relations between the $\mathrm{KP}^{(1)}$ and $\mathrm{KP}^{(m)}$ equations, for $m \geq 3$. As a matter of fact, the existence of such a link between different $\mathrm{KP}^{(m)}$ theories, which gives rise to these mappings of flows, is extremely natural in our approach. It is a consequence of the fact that all $\mathrm{KP}^{(m)}$ theories are different field-theoretic pictures of the same infinite dimensional dynamical system, the central system.

\section{The $\mathrm{KdV}_{n}^{m}$ equation}

The $\mathrm{KdV}_{n}^{m}$ equations are the restriction of the $\mathrm{KP}^{(m)}$ equations to the invariant submanifold $\mathcal{Q}_{n}^{m}$ of the orbits of $X_{m}$ which are tangent to $\mathcal{S}_{n}$ (see Figure 1). In the case $n>m$, the space $\mathcal{Q}_{n}^{m}$ is characterized by the following property.

Proposition 6.1 Let $\left(H^{(1)}, \ldots, H^{(m)}\right)$ be a generic point of $\mathcal{Q}^{m}$, and let $\left(H^{(1)}, \ldots, H^{(n-1)}\right)$ be the first $(n-1)$ currents constructed by the (differential) Fà̀ di Bruno algorithm of Section 5. The point $\left(H^{(1)}, \ldots, H^{(m)}\right)$ belongs to $\mathcal{Q}_{n}^{m}$ if and only if the Laurent series $\left(\partial_{x}+H^{(m)}\right) H^{(\alpha)}$, for $\alpha=0, \ldots, n-1$ admit the linear expansion

$$
\left(\partial_{x}+H^{(m)}\right) H^{(\alpha)}=\sum_{\beta=0}^{n-1} u_{\beta}^{\alpha}(\lambda) H^{(\beta)}
$$


where the coefficients $u_{\beta}^{\alpha}(\lambda)$ depend polynomially on $\lambda=z^{n}$ and on the coordinates $H_{l}^{1}, \ldots, H_{l}^{m}$ of the point of $\mathcal{Q}_{n}^{m}$.

Proof. We recall that the vector field $X_{m}$ is defined by the equation

$$
\left(\frac{\partial}{\partial t_{m}}+H^{(m)}\right)\left(\mathcal{H}_{+}\right) \subset \mathcal{H}_{+}
$$

and that $\mathcal{S}_{n}$ is characterized by the property

$$
z^{n} \cdot\left(\mathcal{H}_{+}\right) \subset \mathcal{H}_{+} \cdot
$$

Thus, on $\mathcal{S}_{n}$ the subspace $\mathcal{H}_{+}$is generated by $\left(H^{(1)}, \ldots, H^{(n-1)}\right)$ and by multiplication by powers of $\lambda$. Equation (6.1) simply means that $X_{m}$ is tangent to $\mathcal{S}_{n}$.

To see how this condition works in practice, we will restrict from now on to the case of $\mathrm{KdV}_{3}^{2}$. In the notations of Section $\Psi$, i.e., $h(z)=H^{(1)}, k(z)=H^{(2)}, \lambda=$ $z^{3}$, the submanifold $\mathcal{Q}_{3}^{2}$ is defined by the constraints

$$
\left(\partial_{x}+k(z)\right)\left[\begin{array}{c}
1 \\
h(z) \\
k(z)
\end{array}\right]=\mathcal{U}(\lambda)\left[\begin{array}{c}
1 \\
h(z) \\
k(z)
\end{array}\right] .
$$

The matrix $\mathcal{U}$ is easily computed by comparing the powers of $z$ on both sides. We obtain the generalized Frobenius matrix

$$
\mathcal{U}=\left(\begin{array}{ccc}
0 & 0 & 1 \\
\lambda+h_{2}+h_{1} & h_{1} & 0 \\
2 k_{2}-h_{3} & \lambda+2 k_{1}-h_{2} & -h_{1}
\end{array}\right) .
$$

Therefore the constraints (6.4) are equivalent to the pair of Riccati equations

$$
\begin{aligned}
& h(z)_{x}+h(z) k(z)=z^{3}+h_{1} h(z)+\left(h_{2}+k_{1}\right) \\
& k(z)_{x}+k(z)^{2}=-h_{1} k(z)+h(z)\left(2 k_{1}-h_{2}+z^{3}\right)-\left(h_{3}-2 k_{2}\right) .
\end{aligned}
$$

Proposition 6.2 The $\mathcal{Q}_{3}^{2}$ constraints allow to express all the fields $\left\{h_{j}, k_{j}\right\}_{j \geq 3}$ of the $K \mathrm{P}^{(2)}$ theory as differential polynomials of the first four components $h_{1}, h_{2}, k_{1}, k_{2}$. Therefore the space $\mathcal{Q}_{3}^{2}$ can be identified with the space of generalized Frobenius matrices (6.5) and $K d V_{3}^{2}$ reduces to a hierarchy on four fields, $h_{1}, h_{2}, k_{1}, k_{2}$. 
Proof. The coefficient of $z^{-1}$ of (6.6) gives

$$
h_{3}=h_{1}^{2}-h_{1 x}-k_{2}
$$

and, in general, the coefficients of $z^{-i}$ of (6.7) and of $z^{-(i+1)}$ of (6.6) can be read as a linear system in the unknowns $\left(k_{i+2}, h_{i+3}\right)$ of the form

$$
\left[\begin{array}{cc}
2 & -1 \\
1 & 1
\end{array}\right]\left[\begin{array}{l}
k_{i+2} \\
h_{i+3}
\end{array}\right]=\left[\begin{array}{l}
\alpha_{i} \\
\beta_{i}
\end{array}\right]
$$

where the RHS is a $x$-differential polynomial in the Laurent coefficients $\left\{h_{l}, k_{m}\right\}$ with $l<i+3, m<i+2$.

To obtain the $\mathrm{KdV}_{3}^{2}$ hierarchy in the form of a zero-curvature equation we consider the vector field $X_{j}$ and the associated current $H^{(j)}$ constructed with the differential Faà di Bruno algorithm of Section 5. Since this vector field is tangent to $\mathcal{S}_{3}$ at the points of $\mathcal{Q}_{3}^{2}$, there exists a matrix $\mathcal{V}^{(j)}(\lambda)$, whose entries $v^{(j)^{\alpha}}(\lambda)$ are polynomials in $\lambda=z^{3}$ with coefficients depending on the Laurent coefficients $\left\{h_{i}\right\}$ and $\left\{k_{i}\right\}$, such that

$$
\left(\frac{\partial}{\partial t_{j}}+H^{(j)}\right) H^{(\alpha)}=\sum_{\beta=0}^{n-1} v_{\beta}^{(j)}{ }_{\beta}^{\alpha}(\lambda) H^{(\beta)}
$$

for $\alpha=0,1,2$.

Proposition 6.3 The $K d V_{3}^{2}$ hierarchy on the space of generalized Frobenius matrices (6.5) admits the zero-curvature representation

$$
\frac{\partial}{\partial t_{j}} \mathcal{U}-\frac{\partial}{\partial x} \mathcal{V}^{(j)}+\left[\mathcal{U}, \mathcal{V}^{(j)}\right]=0
$$

Proof. It is enough to cross differentiate equations (6.1) and (6.10) defining the matrices $\mathcal{U}$ and $\mathcal{V}^{(j)}$, and to recall the result of Proposition 6.2 about the possibility of expressing the Laurent coefficients $h_{j}, k_{j}, j \geq 3$, in terms $\left(h_{1}, h_{2}, k_{1}, k_{2}\right)$.

The same procedure can be used for other fractional KdV equations. It should be compared with the approach of $[8,9]$. 


\section{On the Bihamiltonian aspects of $\mathrm{KdV}_{3}^{2}$}

To show that our $\mathrm{KdV}_{3}^{2}$ hierarchy coincides with the fractional $\mathfrak{s l}_{3}^{(2)} \mathrm{KdV}$ hierarchy of [25], it could suffice to remark that $\mathrm{KdV}_{3}^{2}$ can be also obtained from $\mathrm{KdV}_{3}^{1}$ (which is the classical Boussinesq hierarchy) by means of the $x \leftrightarrow t_{2}$ interchange, in the same way as $\mathrm{KP}^{(2)}$ is obtained from $\mathrm{KP}^{(1)}$. Actually, this hierarchy was first studied in [7] by means of the Hamiltonian reduction suggested in [26, 6], and the existence of two compatible Hamiltonian structures was also pointed out. The theory of fractional KdV hierarchy has been further explored and generalized in a number of papers (see, e.g., [8, 9, 11, 12]), by means of the study of its Lie-algebraic aspects.

In this section we will briefly address the problem of showing a direct relation between the $\mathrm{KdV}_{3}^{2}$ treated in Section 6 and the corresponding generalized $\mathfrak{s l}_{3}^{(2)}$ DS one of [7, 8], assuming as a starting point its bihamiltonian aspects. Full proofs and detailed explanations will be given elsewhere [27]. The reader should keep in mind the logical path followed in Section 2, where the $\mathrm{KP}^{(1)}$ hierarchy has been derived from the Poisson pencil of KdV.

We recall that a manifold $\mathcal{M}$ is said to be bihamiltonian if it is endowed with two compatible Poisson brackets, and that a Poisson bracket can be assigned in terms of a Poisson tensor $P: T^{*} \mathcal{M} \rightarrow T \mathcal{M}$ as

$$
\{f, g\}=\langle d f, P d g\rangle \text {. }
$$

We consider the Lie algebra $\mathfrak{s l}(3)$ and its loop algebra $\mathfrak{G}=L(\mathfrak{s l}(3))$, i.e., the space of $C^{\infty}$-maps from $S^{1}$ to $\mathfrak{s l}(3)$. The algebra $\mathfrak{G}$ is a bihamiltonian manifold (see, e.g., [3, 20]). The Poisson structures we will consider hereinafter are:

$$
\begin{aligned}
& \left(P_{0}\right)_{M} \cdot V=\left[A_{2}, V\right] \\
& \left(P_{1}\right)_{M} \cdot V=V_{x}+[V, M] .
\end{aligned}
$$

Here, $M$ is a point in $\mathfrak{G}, V$ is a cotangent vector at $M, x$ is the parameter on $S^{1}$, and $A_{2}$ is the element

$$
A_{2}=\left(\begin{array}{lll}
0 & 0 & 0 \\
1 & 0 & 0 \\
0 & 1 & 0
\end{array}\right)
$$

in $\mathfrak{G}$. To lower the number of degrees of freedom, one seeks (following the seminal paper [3]) a Poisson reduction of this bihamiltonian structure. According to the bihamiltonian variant [20] of the Marsden-Ratiu reduction theorem [28], one has to consider a symplectic leaf $\mathcal{S}$ of $P_{0}$, the (integrable) distribution 
$D=P_{1}\left(\right.$ ker $\left.P_{0}\right)$, and the intersection $E=D \cap T \mathcal{S}$. Then the quotient manifold $\mathcal{N}=\mathcal{S} / E$ is still a bihamiltonian manifold. In our case, we choose $\mathcal{S}$ to be the symplectic leaf passing through the point

$$
B_{2}=\left(\begin{array}{ccc}
0 & 0 & 1 \\
0 & 0 & 0 \\
0 & 0 & 0
\end{array}\right)
$$

The submanifold $\mathcal{S}$ has dimension 6 over $C^{\infty}\left(S^{1}\right)$, and its generic point can be parametrized as

$$
S=\left(\begin{array}{ccc}
p_{0}(x) & p_{2}(x) & 1 \\
q_{0}(x) & p_{1}(x)-p_{0}(x) & -p_{2}(x) \\
q_{2}(x) & q_{1}(x) & -p_{1}(x)
\end{array}\right) .
$$

Then we consider the distribution $D$, which turns out to be tangent to $\mathcal{S}$, so $E=D$. The quotient manifold $\mathcal{N}=\mathcal{S} / E$ is parametrized by the four fields

$$
\begin{aligned}
& u_{0}=-p_{0}+p_{1}+p_{2}^{2} \\
& u_{1}=q_{0}+2 p_{0} p_{2}-p_{1} p_{2}-p_{2}^{3}+p_{2 x} \\
& u_{2}=q_{1}-p_{0} p_{2}+2 p_{1} p_{2}+p_{2}^{3}+p_{2 x} \\
& u_{3}=q_{2}+p_{0 x}-p_{2} p_{2 x}+q_{0} p_{2}-q_{1} p_{2}+p_{0} p_{1}-p_{2}^{4}-2 p_{1} p_{2}^{2} .
\end{aligned}
$$

The reduced Poisson pencil $P_{\lambda}^{\mathcal{N}}=P_{1}^{\mathcal{N}}-\lambda P_{0}^{\mathcal{N}}$ on $\mathcal{N}$ turns out to be

$$
P_{\lambda}^{\mathcal{N}}=\left(\begin{array}{cccc}
\frac{2}{3} \partial_{x} & -u_{1}-\lambda & u_{2}+\lambda & \left(\frac{1}{3} \partial_{x}^{2}-u_{0} \partial_{x}-u_{0 x}\right) \\
* & 0 & \left(P_{\lambda}^{\mathcal{N}}\right)^{23} & \left(2 u_{1} \partial_{x}+u_{1 x}-2 u_{0} u_{1}\right)-\lambda\left(-2 \partial_{x}+2 u_{0}\right) \\
* & * & 0 & \left(u_{2} \partial_{x}+u_{2 x}+2 u_{0} u_{2}\right)-\lambda\left(-2 \partial_{x}-2 u_{0}\right) \\
* & * & * & \left(P_{\lambda}^{\mathcal{N}}\right)^{44}
\end{array}\right),
$$

where

$$
\begin{aligned}
& \left(P_{\lambda}^{\mathcal{N}}\right)^{23}=-\partial_{x}^{2}+3 u_{0} \partial_{x}+2 u_{0 x}+u_{3}-2 u_{0}^{2} \\
& \left(P_{\lambda}^{\mathcal{N}}\right)^{44}=-\frac{2}{3} \partial_{x}^{3}+\left(\frac{4}{3} u_{0 x}+2 u_{3}+\frac{2}{3} u_{0}^{2}\right) \partial_{x}+\frac{2}{3} u_{0} u_{0 x}+u_{3 x}+\frac{2}{3} u_{0 x x} .
\end{aligned}
$$


They are computed according to the standard procedure explained in [28, 20]. The reduced Poisson tensor $P_{1}^{\mathcal{N}}$ coincides with the one given in [9], after the change of coordinates

$$
u_{0}=-\tilde{U}, \quad u_{1}=\tilde{G}^{+}, \quad u_{2}=\tilde{G}^{-}, \quad u_{3}=\tilde{T}-\tilde{U}^{2}+\frac{1}{2} \tilde{U}_{x}
$$

and $x \mapsto-x$.

The basic remark to connect this theory with the $\mathrm{KdV}_{3}^{2}$ treated in Section 6 is that the quotient space $\mathcal{N}$ can be identified with the space of (generalized) Frobenius matrices

$$
\mathcal{U}=\left(\begin{array}{ccc}
0 & 0 & 1 \\
u_{1} & u_{0} & 0 \\
u_{3} & u_{2} & -u_{0}
\end{array}\right)
$$

by noticing that the constraints $p_{0}=p_{2}=0$ define a submanifold $U$ of $\mathcal{S}$ transversal to the distribution $D$. Consequently, by comparing the matrices $(6.5)$ and $(7.10)$ we arrive at the identification

$$
\begin{gathered}
u_{0}=h_{1}, \quad u_{1}=h_{2}+k_{1}, \quad u_{2}=2 k_{1}-h_{2} \\
u_{3}=2 k_{2}-h_{3}=3 k_{2}-h_{1}^{2}+h_{1 x},
\end{gathered}
$$

which can be inverted in local form to yield

$$
\begin{aligned}
& h_{1}=u_{0}, \quad h_{2}=\frac{1}{3}\left(2 u_{1}-u_{2}\right) \\
& k_{1}=\frac{1}{3}\left(u_{1}+u_{2}\right), \quad k_{2}=\frac{1}{3}\left(u_{3}+u_{0}^{2}-u_{0 x}\right) .
\end{aligned}
$$

This procedure sets up a diffeomorphism

$$
\Phi: \mathcal{N} \longrightarrow \mathcal{Q}_{3}^{2}
$$

between the reduced bihamiltonian manifold $\mathcal{N}$ and the phase space of the $\mathrm{KdV}_{3}^{2}$ theory. This diffeomorphism enjoys the following properties, which we limit ourselves to state without proofs (they will be detailed elsewhere [27]):

1. The integral on $S^{1}$

$$
H(z)=3 z^{2} \int_{S^{1}} k(z) d x
$$

of the pull-back of the second current of the $\mathrm{KdV}_{3}^{2}$ theory is a Casimir function of the Poisson pencil (7.8).

2. The push-forward of the Hamiltonian vector fields on $\mathcal{N}$ associated with this Casimir function are the $\mathrm{KdV}_{3}^{2}$ flows 6.11) on $\mathcal{Q}_{3}^{2}$. Hence, we are allowed to identify the two theories. 
This result can be usefully compared with the KdV case briefly treated in Section 2. The $\operatorname{KdV}\left(=\mathrm{KdV}_{2}^{1}\right)$ theory is a theory in a single field $u$, defined on the quotient space $\mathcal{N}_{2}^{1}$ associated with the Lie-Poisson pencil on the loop algebra of $\mathfrak{s l}_{2}$ and with the matrices [20]

$$
A_{1}=\left(\begin{array}{cc}
0 & 0 \\
1 & 0
\end{array}\right) \quad B_{1}=\left(\begin{array}{cc}
0 & 1 \\
0 & 0
\end{array}\right) .
$$

The $\mathrm{KdV}_{3}^{2}$ theory is a theory on four fields, $\left(u_{0}, u_{1}, u_{2}, u_{3}\right)$ defined on the quotient space $\mathcal{N}_{3}^{2}$ associated with the Lie-Poisson pencil on the loop algebra of $\mathfrak{s l}_{3}$ and with the matrices

$$
A_{2}=\left(\begin{array}{ccc}
0 & 0 & 0 \\
1 & 0 & 0 \\
0 & 1 & 0
\end{array}\right) \quad B_{2}=\left(\begin{array}{ccc}
0 & 0 & 1 \\
0 & 0 & 0 \\
0 & 0 & 0
\end{array}\right) .
$$

In the first case the Casimir function of the reduced Poisson pencil is defined by the solution $h(z)$ of the single Riccati equation

$$
h_{x}+h^{2}=u+z^{2} .
$$

In the latter case, the Casimir function is computed by solving the pair of Riccati equations

$$
\begin{aligned}
& h_{x}+k h=u_{0} h+\left(u_{1}+\lambda\right) \\
& k_{x}+k^{2}=-u_{0} k+\left(u_{2}+\lambda\right) h+u_{3},
\end{aligned}
$$

in the first two currents $H^{(1)}=h(z), H^{(2)}=k(z)$. The appearance of these systems of Riccati equations is a general feature of $\mathrm{KdV}_{m}^{n}$ theories with $m \geq 2$ which, in our opinion, deserves further attention.

When the point $u$ evolves according to the KdV hierarchy, the solution $h$ of the Riccati equation (7.17) evolves according to the $\mathrm{KP}^{(1)}$ equations

$$
\frac{\partial}{\partial t_{j}} h=-\pi_{-}\left(h H^{(j)}\right)
$$

defined in Section 2. Similarly, when the point $\left(u_{0}, u_{1}, u_{2}, u_{3}\right)$ evolves according to the $\mathrm{KdV}_{3}^{2}$ hierarchy, the solution $(h, k)$ of the Riccati system (7.18) evolves according to the $\mathrm{KP}^{(2)}$ equations

$$
\begin{aligned}
\frac{\partial}{\partial t_{j}} h & =-\pi_{-}\left(h H^{(j)}\right) \\
\frac{\partial}{\partial t_{j}} k & =-\pi_{-}\left(k H^{(j)}\right)
\end{aligned}
$$


In both cases the current densities $H^{(j)}$, which are constructed in a different way in the two theories evolve in time according to the central system (1.2). This completes the view of the relations connecting the central system and the fractional $\mathrm{KdV}$ hierarchies we discussed in the case of $\mathrm{KdV}_{3}^{2}$.

\section{References}

[1] V. A. Fateev, S. L. Lukyanov, Additional symmetries and exactly-soluble models in two-dimensional conformal field theory. Sov. Sci. Rev. A. Phys. 15 (1990), 1-124.

[2] T. G. Khovanova, The Gel'fand-Dikii Lie Algebras and the Virasoro Algebra. Funct. Anal. Appl. 20 (1987), 332-334.

[3] V. G. Drinfeld, V. V. Sokolov, Lie Algebras and Equations of Korteweg-de Vries Type. J. Sov. Math. 30 (1985), 1975-2036.

[4] L. A. Dickey, Soliton Equations and Hamiltonian Systems. Adv. Series in Math. Phys. Vol. 12, World Scientific, Singapore, 1991.

[5] P. Bouwknegt and K. Schoutens (eds.), W-symmetry. Adv. Series in Math. Phys. Vol. XX, World Scientific, Singapore, 1995.

[6] M. Bershadsky, Conformal Field Theories via Hamiltonian reductions. Comm. Math. Phys. 139 (1991), 71-82.

[7] I. Bakas, D. A. Depireux, A Fractional KdV Hierarchy. Mod. Phys. Lett A6 (1991), 1561-1573.

[8] M. F. de Groot, T. J. Hollowood, J. L. Miramontes, Generalized Drinfel'dSokolov Hierachies. Comm. Math. Phys. 145 (1992), 57-84.

[9] N. J. Burroughs, M. F. DeGroot, T. J. Hollowood, J. L. Miramontes, Generalized Drinfel'd-Sokolov Hierachies. II. The Hamiltonian structures. Comm. Math. Phys. 153 (1993), 187-215.

[10] D. A. Depireux, P. Mathieu, On the Classical $W_{N}^{(l)}$ algebras. Int. Jour. Mod. Phys. A7 (1992), 6053-6080.

[11] L. Fehér, J. Harnad, I. Marshall, Generalized Drinfeld-Sokolov Reductions and KdV type Hierarchies. Comm. Math. Phys. 154 (1993), 181-214.

[12] C. R. Fernández-Pousa, M. V. Gallas, J. L. Miramontes, J. Sánchez Guillen, $W$-Algebras from soliton equations and Heisenberg subalgebras. Ann. Phys. 243 (1995), 372-419.

[13] G. Segal, G. Wilson, Loop Groups and equations of the KdV type. Publ. Math. IHES, 61 (1985), 5-65.

[14] E. Date, M. Jimbo, M. Kashiwara, T. Miwa, Transformation Groups for Soliton Equations. Proceedings of R.I.M.S. Symposium on Nonlinear Integrable Systems-Classical Theory and Quantum Theory, M. Jimbo, T. Miwa eds., 39-119, World Scientific, Singapore, 1983. 
[15] M. Sato, Y. Sato, Soliton equations as dynamical systems on infinitedimensional Grassmann manifold. Nonlinear PDEs in Applied Sciences (US-Japan Seminar, Tokyo), P. Lax and H. Fujita eds., 259-271, NorthHolland, Amsterdam, 1982.

[16] G. Falqui, F. Magri, M. Pedroni, Darboux Transformations and Linearization of the KP Hierarchy. In preparation (1997).

[17] O. I. Bogoyavlensky, S. P. Novikov, The relationship between Hamiltonian formalism of stationary and non-stationary problems. Funct. Anal. Appl. 10 (1976), 92-95.

[18] B. A. Dubrovin, V. B. Matveev, S. P. Novikov, Non-linear equations of the Korteweg-de Vries type, finite-zone linear operators, and Abelian varieties. Russ. Math. Surv. 31 (1976), 59-146.

[19] I. M. Gelfand, I. Zakharevich, On the local geometry of a bi-Hamiltonian structure. In The Gelfand Mathematical Seminars 1990-1992 (L. Corwin et al. eds.), pp. 51-112, Birkäuser, Boston, 1993.

[20] P. Casati, F. Magri, M. Pedroni, Bihamiltonian Manifolds and $\tau$-function. In: Mathematical Aspects of Classical Field Theory 1991 (M. J. Gotay et al. eds.), Contemporary Mathematics, Vol. 132, 213-234, American Mathematical Society, Providence, R. I., 1992.

[21] P. Casati, G. Falqui, F. Magri, M. Pedroni, a) The KP theory revisited. II. The Reduction Theory and the Adler-Gel'fand-Dickey brackets. SISSA preprint 3/96/FM. b) The KP theory revisited. III. The Bihamiltonian Action and Gel'fand-Dickey equations. SISSA preprint 4/96/FM.

[22] G. Wilson, On two Constructions for Conservation Laws for Lax equations. Quart. J. Math. Oxford 32 (1981), 491-512.

[23] P. Casati, G. Falqui, F. Magri, M. Pedroni, The KP theory revisited. IV. KP equations, Dual KP equations, Baker-Akhiezer and $\tau$-functions. SISSA preprint 5/96/FM.

[24] F. Magri, M. Pedroni, J. P. Zubelli, On the Geometry of Darboux Transformations for the KP Hierarchy and its Connection with the Discrete KP Hierarchy. To appear in Comm. Math. Phys. (1997).

[25] P. Mathieu, W. Oevel, The $W_{3}^{(2)}$ conformal algebra and the Boussinesq hierarchy. Mod. Phys. Lett. A6 (1991), 2397-2404.

[26] A. M. Polyakov, Gauge Transformations and Diffeomorphisms. Int. Jour. Mod. Phys. A5 (1990), 833-850.

[27] P. Casati, G. Falqui, F. Magri, M. Pedroni, A note on fractional KdV hierarchies. II. In preparation.

[28] J. E. Marsden, T. Ratiu, Reduction of Poisson Manifolds. Lett. Math. Phys. 11 (1986), 161-169. 